\title{
Development of EUROFER97 Database and Material Property Handbook
}

E. Gaganidze ${ }^{1}$, F. Gillemot ${ }^{2}$, I. Szenthe ${ }^{2}$, M. Gorley ${ }^{3}$, M. Rieth ${ }^{1}$, E. Diegele ${ }^{4 \& 1}$

${ }^{1}$ Karlsruhe Institute of Technology, Institute for Applied Materials, Germany

2MTA Centre for Energy Research, Hungary

${ }^{3}$ Culham Centre for Fusion Energy, UK

${ }^{4}$ EUROfusion PMU, Germany

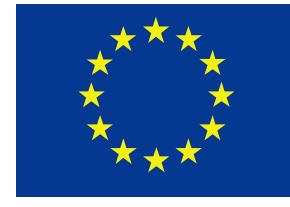

This work has been carried out within the framework of the EUROfusion Consortium and has received funding from the Euratom research and training programme 2014-2018 under grant agreement No 633053. The views and opinions expressed herein do not necessarily reflect those of the European Commission. 


\section{EUROfusion Material Database \& Handbook (MD\&H)}

Realization of in-vessel components for DEMO

- Development of sound material databases (MD) on structural, armour and functional materials

Development of DEMO Design Criteria (DDC) for in-vessel components

\section{EUROfusion / PPPT / WPMAT}

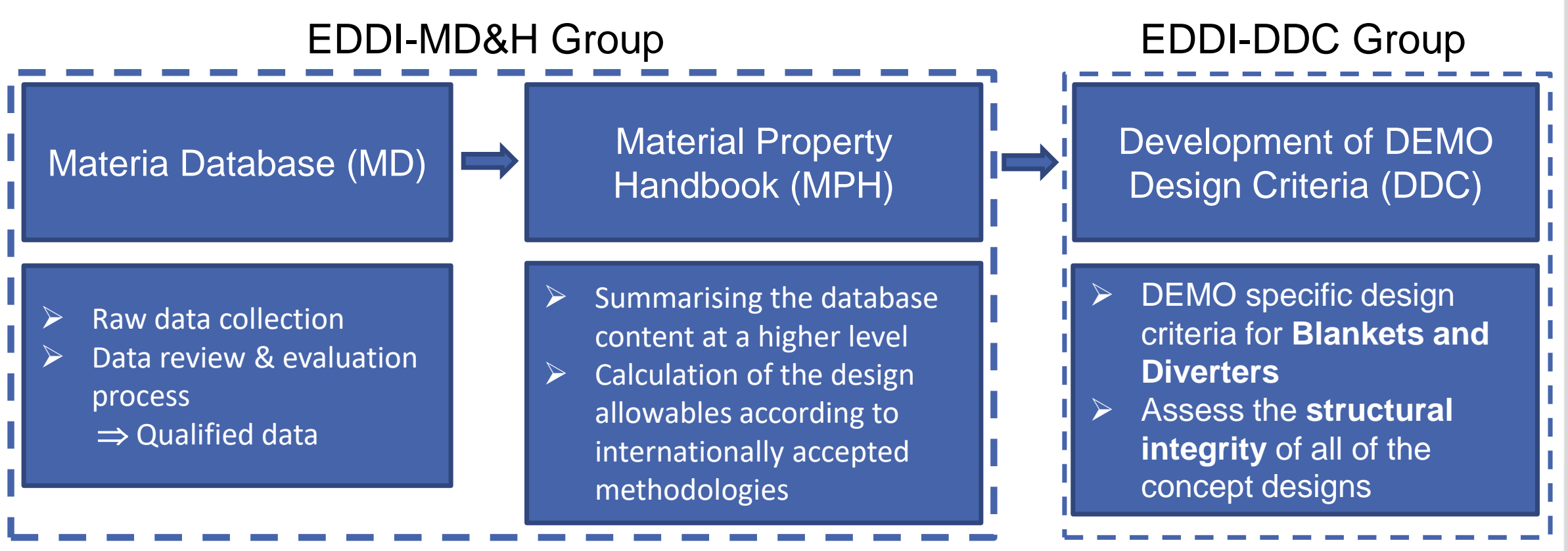

Objective: Development of Material Database \& Material Property Handbook on Reduced Activation Ferritic/Martensitic steel EUROFER97
$2 \quad 6.11 .2017$
E. Gaganidze

EUROfusion 


\section{European Reference RAFM Steel for ITER TBM and DEMO EUROFER 97}

Chemical composition - Main alloying elements (targeted values in [])

\begin{tabular}{|c|c|c|c|c|c|c|c|c|c|c|c|}
\hline & $\mathrm{C}$ & $\mathrm{Cr}$ & $\mathrm{W}$ & $\mathrm{Mn}$ & $\mathrm{V}$ & $\mathrm{Ta}$ & $\mathrm{N} 2$ & $\mathrm{P}$ & $\mathrm{S}$ & $\mathrm{B}$ & $\mathrm{O} 2$ \\
\hline Specified (mass\%) & $\begin{array}{c}0.09-0.12 \\
{[0.11]}\end{array}$ & $\begin{array}{c}8.5-9.5 \\
{[9.0]}\end{array}$ & $\begin{array}{c}1.0-1.2 \\
{[1.1]}\end{array}$ & $\begin{array}{c}0.20-0.60 \\
{[0.40]}\end{array}$ & $0.15-0.25$ & $\begin{array}{c}0.10-0.14 \\
{[0.12]}\end{array}$ & $\begin{array}{c}0.015-0.045 \\
{[0.030]}\end{array}$ & $<0.005$ & $<0.005$ & $<0.001$ & $<0.01$ \\
\hline Achieved (mass\%) & $0.11-0.12$ & $8.82-8.96$ & $1.07-1.15$ & $0.38-0.49$ & $\begin{array}{c}0.018- \\
0.034\end{array}$ & $0.13-0.15$ & $0.018-0.034$ & $\begin{array}{r}0.004- \\
0.005\end{array}$ & $\begin{array}{c}0.003- \\
0.004\end{array}$ & $\begin{array}{c}0.0005- \\
0.0009\end{array}$ & $\begin{array}{c}0.0013- \\
0.0018\end{array}$ \\
\hline
\end{tabular}

Chemical composition - Radiologically undesired elements

\begin{tabular}{|l|c|c|c|c|c|c|c|c|}
\hline & $\mathrm{Nb}$ & $\mathrm{Mo}$ & $\mathrm{Ni}$ & $\mathrm{Cu}$ & $\mathrm{Al}$ & $\mathrm{Ti}$ & $\mathrm{Si}$ & $\mathrm{Co}$ \\
\hline Specified $(\mu \mathrm{g} / \mathrm{g})$ & $<10$ & $<50$ & $<50$ & $<50$ & $<100$ & $<100$ & $<500$ & $<50$ \\
\hline Achieved $(\mu \mathrm{g} / \mathrm{g})$ & $2-7$ & $10-32$ & $70-280$ & $15-220$ & $60-90$ & $50-90$ & $400-700$ & 30-70 R. Lindau, Fus. Eng. Des. 75-79 (2005) \\
\hline
\end{tabular}

- Production of large industrial batches: EUROFER 97-1, EUROFER 97-2, EUROFER97-3

- Availability of different product forms: plates, rods, tubes

- Examination \& characterization by several European Research Units (RUs)

- Development of joining technologies

- Investigation of irradiation influence 


\section{EUROFER data source for Material Database}

\section{- Data generated by participant RUs in the course of EFDA/EUROfusion Work Programmes}

- Data published in the open literature

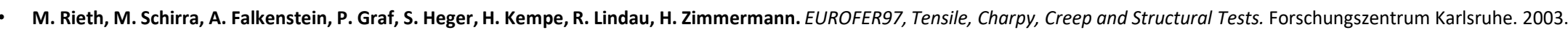
Wissenschaftliche Berichte. FZKA 6911.

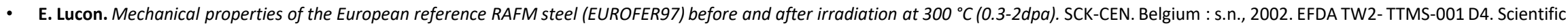
Report SCK.CEN-BLG-962.

- J. Rensman. NRG irradiation testing: Report on $300^{\circ} \mathrm{C}$ and $60^{\circ} \mathrm{C}$ irradiated RAFM. Nuclear Research and consultancy Group (NRG). The Netherlands : s.n., 2005. 20023/05.68497/P.

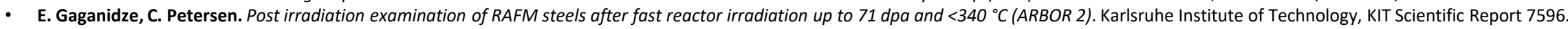
2011.

- E. Lucon, W. Vandermeulen. Overview and Critical Assessment of the Tensile Properties of unirradiated and irradiated EUROFER97. 2007. Open report SCK.CEN-BLG-1042.

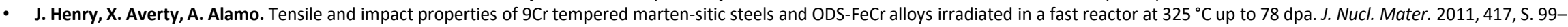
103.

- C. Petersen. Post irradiation examination of RAF/M steels after fast reactor irradiation up to 33 dpa and $<340^{\circ} \mathrm{C}$ (ARBOR 1). Karlsruher Institut für Technologie. 2010. FZKA 7517.

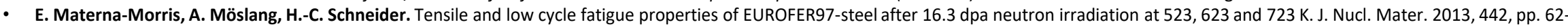
66.

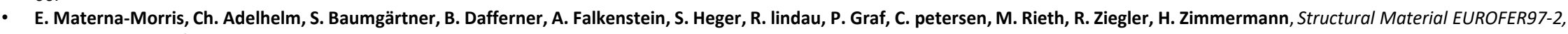
Characterization of $100 \mathrm{~mm}$ Rod Material: Structural, Tensile, Charpy, and Creep Properties, Forschungszentrum Karlsruhe, 2006.

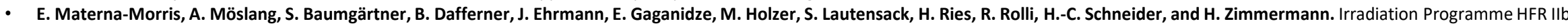

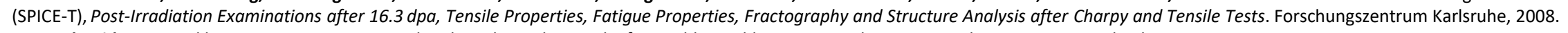

- H.-C. Schneider. Entwicklung einer miniaturisierten bruchmechanischen Probe für Nachbestrahlungsuntersuchungen. Forschungszentrum Karlsruhe. Germany, 2005. FZKA 7066.

- N. Ilchuk, P. Spätig, G.R. Odette. Fracture toughness characterization in the lower transition of neutron irradiated Eurofer97 steel. J. Nucl. Mater. 2013, Vol. 442, pp. 58-61.

- M. Kytka, M. Brumovsky, M. Falcnik. Irradiation embrittlement characterization of the EUROFER 97 material. J. Nucl. Mater. 2011, Bd. 409, S. 147-152.

- R. Chaouadi. Effect of irradiation-induced plastic flow localization on ductile crack resistance behavior of a 9\%Cr tempered martensitic steel. J. Nucl. Mater. 2006, 372, S. 379-390.

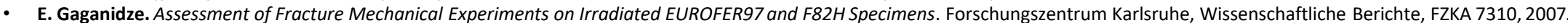

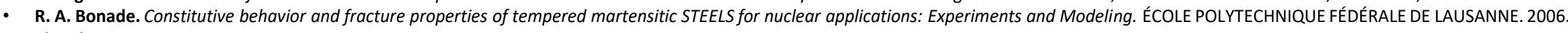
PhD Thesis.

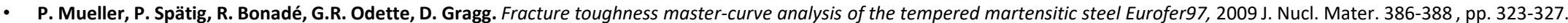

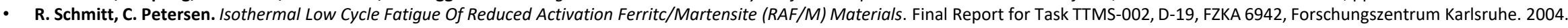

- P. Marmy. Low cycle fatigue and creep-fatigue of Eurofer97. Ecole Polytechnique fédérale de Lausanne. Switzerland : s.n., 2006. LRP 826/06.

- P. Fernandez. LCF Properties of EUROFER. CIEMAT. 2014. private communication.

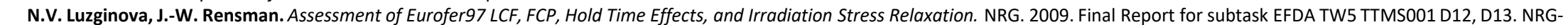
21641/09.95503/E.

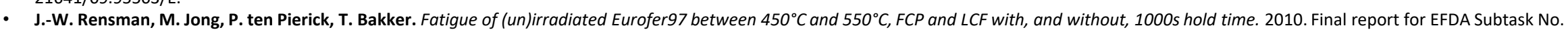
TW2-TTMS-001b-D1. NRG-20903/10.105274.

- M. Rieth, S. Hegger, Creep Properties of EUROFER97. KIT. private communication. 2015

- F. Tavassoli, Comparison of 316L(N) -IG and Eurofer for Early DEMO, EFDA-WP13-MAT-02-01., 2013

\section{EUROFER97 database accounting 3000 data records}

$4 \quad 6.11 .2017 \quad$ E. Gaganidze




\section{Data qualification}

\section{Data review against Data Quality Thresholds}

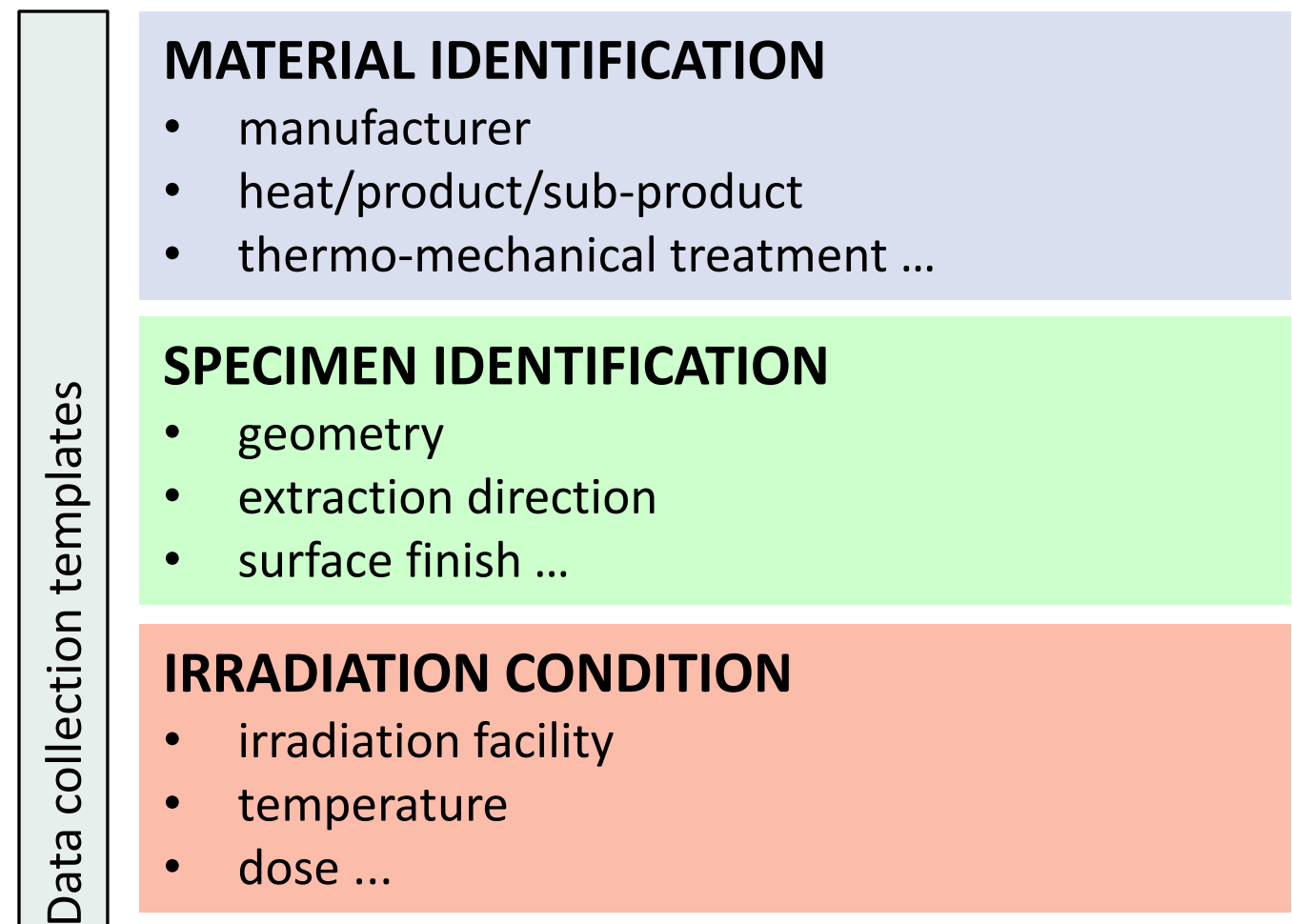

\section{TESTING and RESULTS}

- testing standard, parameter

- temperature

- environment

- results, validity ... 


\section{Data qualification}

\section{Tensile properties}
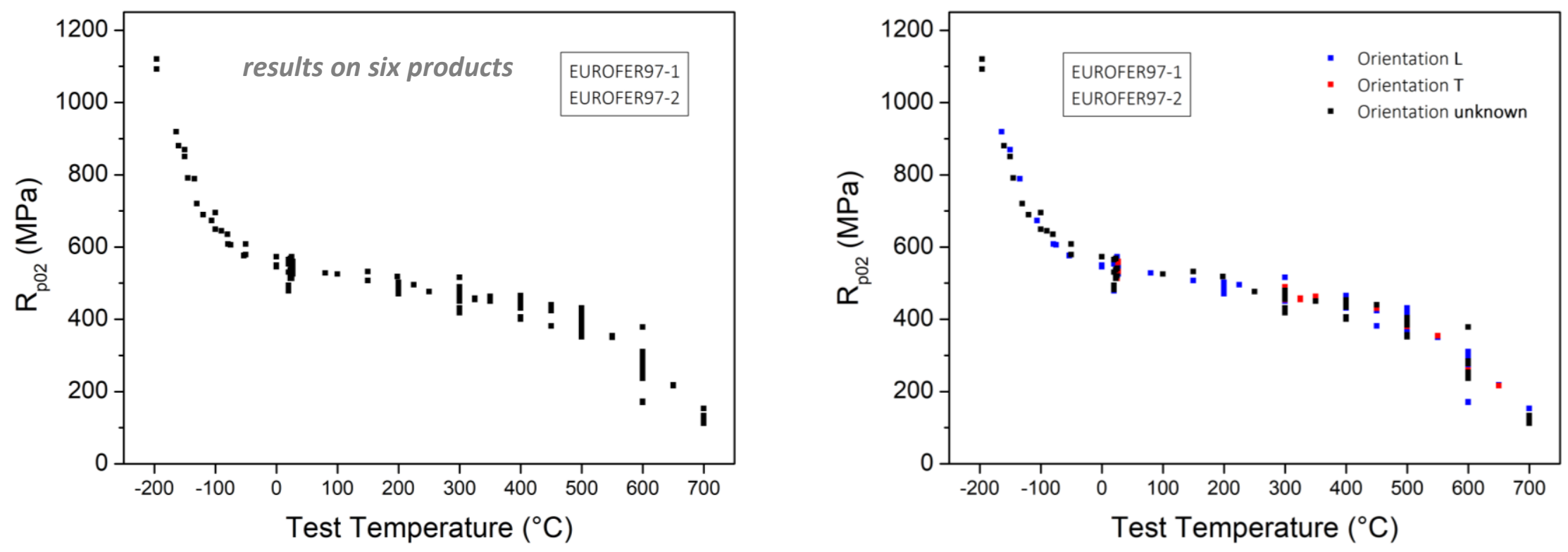

\section{Approach for MPH}

- No differentiation between different heats

- No differentiation between different orientations

- Thermally aged data excluded from allowable calculation

- Irradiation data excluded from allowable calculation 


\section{Data qualification LCF properties}

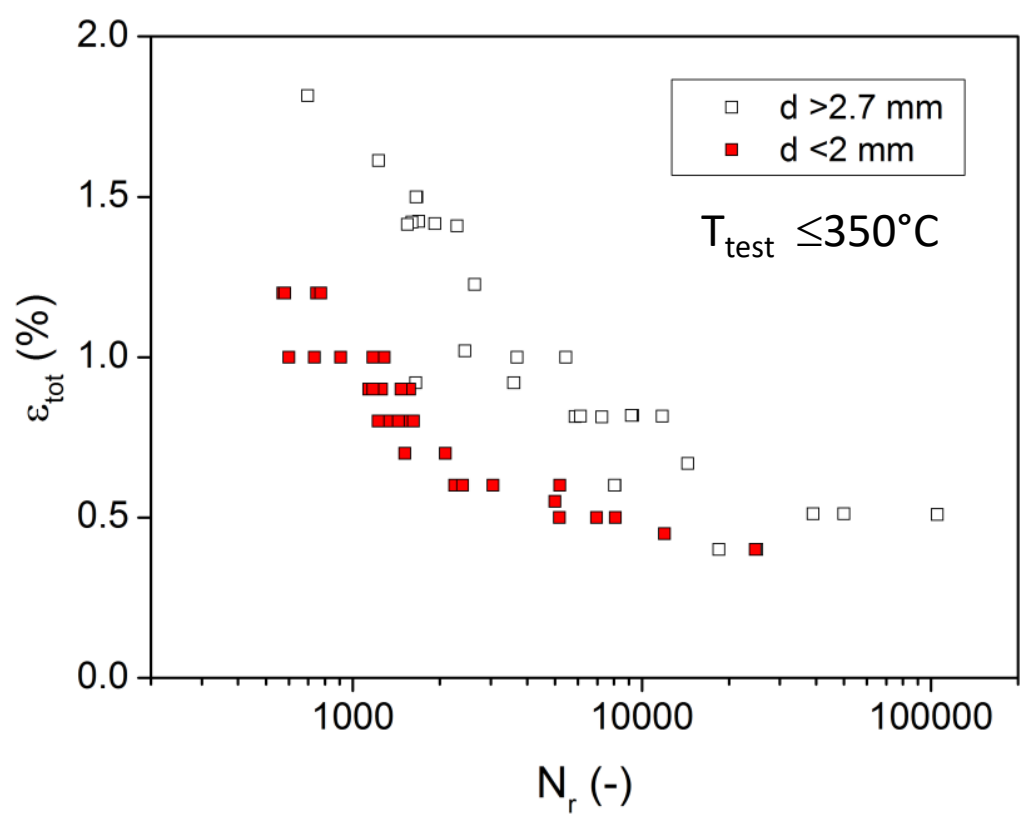

\section{SSTT vs. large specimen}

Considerable underestimation of fatigue lifetime in the reference unirradiated state by SSTT

\section{Approach for MPH}

- No differentiation between different heats

- No differentiation between different orientations

- Differentiation between 'small' and 'large' specimens 


\section{Data qualification \\ Thermal creep properties}

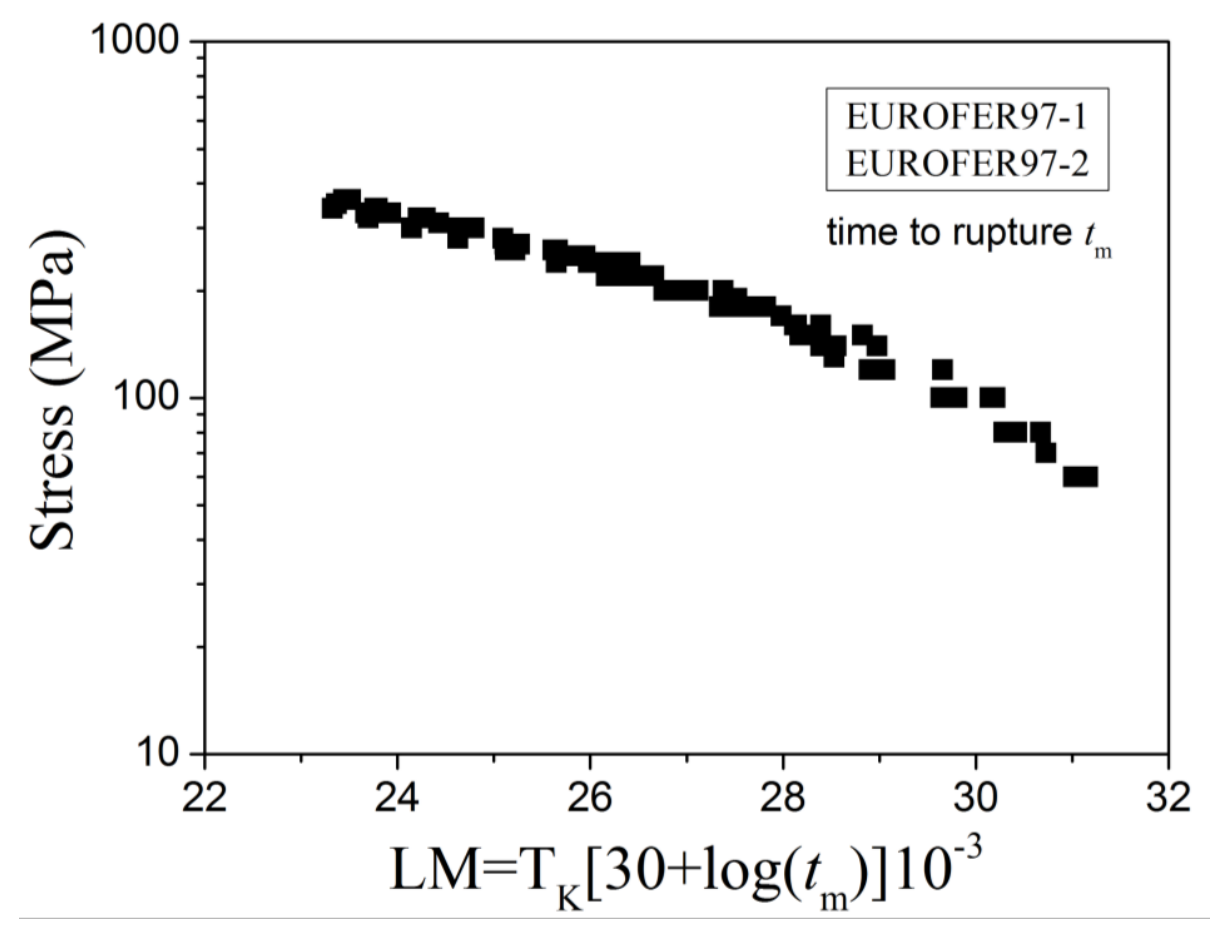

Approximately 140 original creep curves analysed with respect to the design relevant parameters

- Onset of the tertiary creep

- Time to reach $1 \%$ total strain

- Time to reach $0.05 \%$ creep strain

- ...

\section{Approach for MPH}

- No differentiation between different heats 


\section{Allowable calculation}

\section{Average and minimum Yield Strength at $0.2 \%$ offset}

\section{RCC-MRx}

- The average values determined by a statistical method

- Determination of the ratio minimum yield strength at RT to average yield strength at RT

$$
\begin{aligned}
& R_{p 02, \min (R C C-M R x,)}(T) \\
= & \frac{R_{p 02, \min }(R T)}{R_{p 02, a v}(R T)} R_{p 02, a v}(T)
\end{aligned}
$$

\section{ITER SDC-IC}

- Calculate an average $S_{y, a v}$ curve by a statistical method

- Built the minimum curve according to ITER SDC-IC

$$
S_{y, \min }(T)=S_{y, a v}(T)-1.96 \sigma
$$




\section{Material Property Handbook - Pilot project on EUROFER97}

\section{Content}

1. SHORT INTRODUCTION AND OBJECTIVES OF WORK, CHANGES FROM THE LAST ISSUE ............... 6

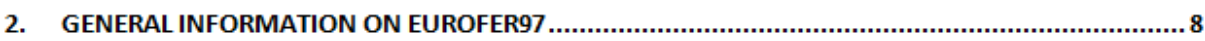

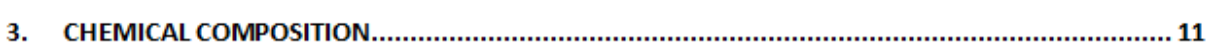

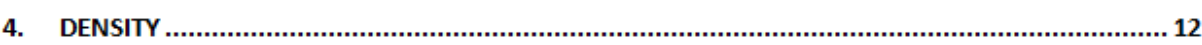

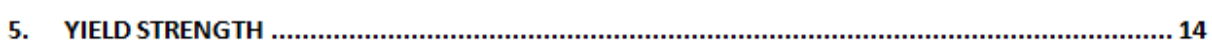

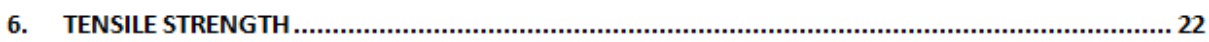

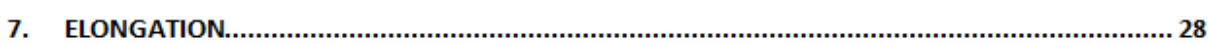

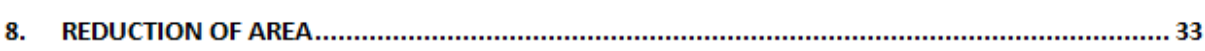

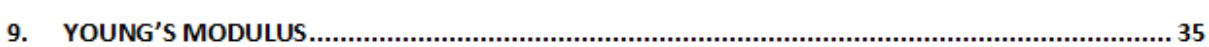

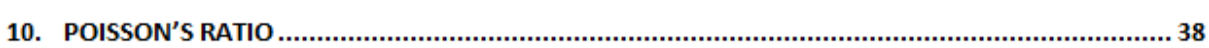

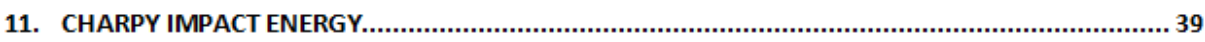

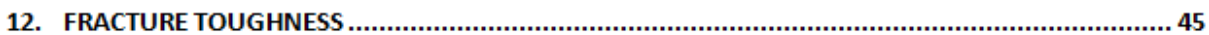

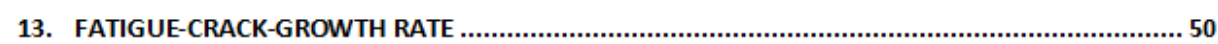

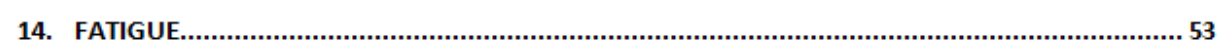

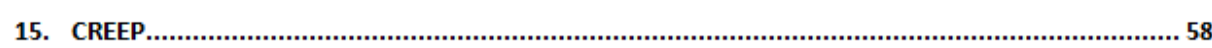

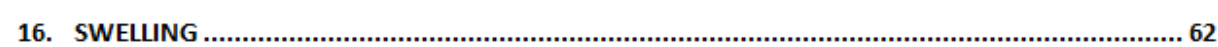

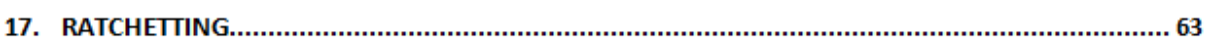

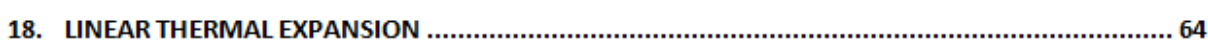

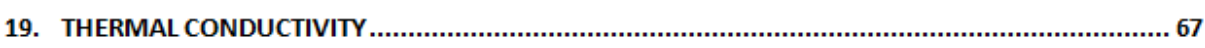

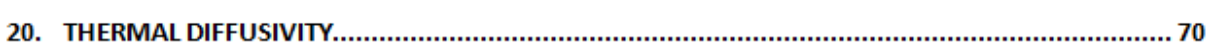

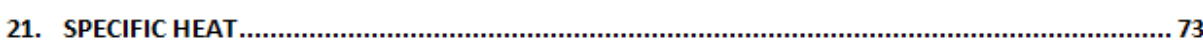

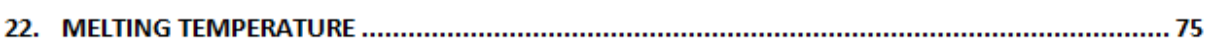

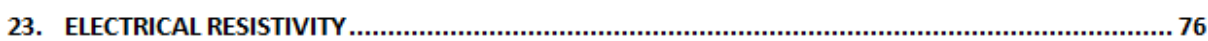

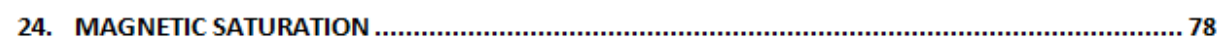

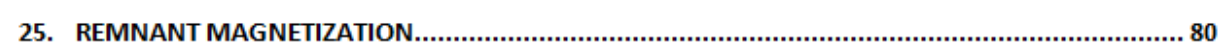

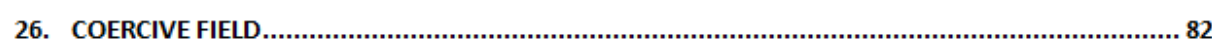

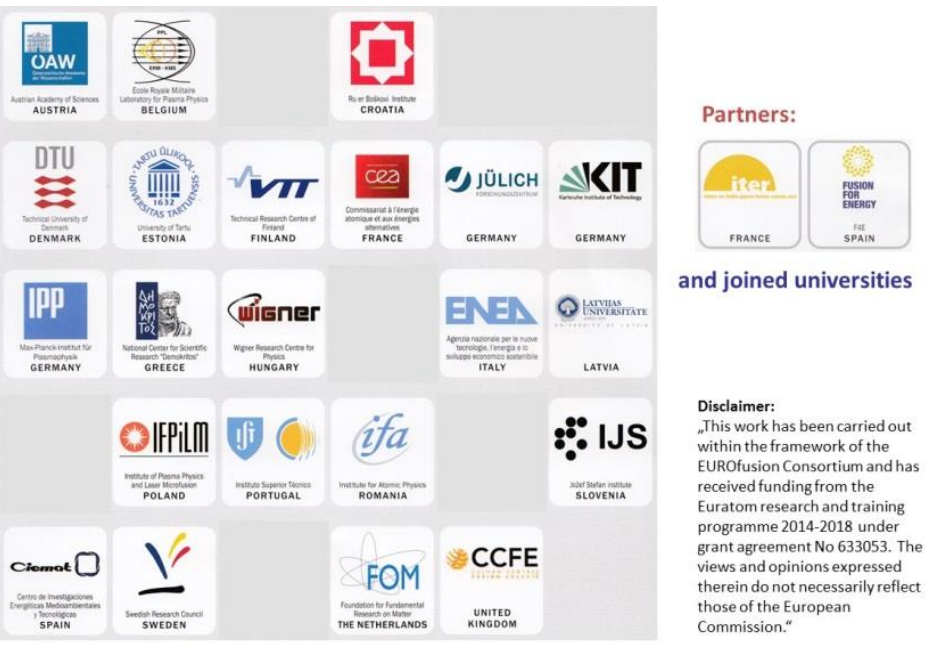

Over 20 DEMO design relevant material properties on base metal EUROFER97 


\section{Material EUROFER \\ Property Composition}

\section{EUROfusion}

POWER PLANT PHYSICS \& TECHNOLOGY DEPARTMENT

demo Material Properties Handbook

\begin{tabular}{|l|l|}
\hline MATERIAL & PROPERTY \\
EUROFER97 & 4.COMPOSITION \\
\hline
\end{tabular}

4. Chemical composition

Standard nominal composition

EUROFER97 presently is a research material under development for use in nuclear and fusion facilities. The composition of the presently tested heat is given in table 4.1. [1] This analyses may slightly diffe from the present and future composition given by the nuclear codes. In case of documents preparation for the authority please check the relevant code.

Table 4.1. The chemical composition of EUROFER97 in wt \%

\begin{tabular}{|c|c|c|c|c|c|c|c|c|c|}
\hline & $\mathrm{C} \%$ & $\mathrm{~S} \%$ & $\mathrm{P} \%$ & $\mathrm{Si} \%$ & $\mathrm{Mn} \%$ & $\mathrm{Ni} \%$ & $\mathrm{Cr} \%$ & $\mathrm{Mo} \%$ & $\mathrm{~W} \%$ \\
\hline $\min$ & 0.09 & - & - & - & 0.20 & - & 8.5 & - & 1.00 \\
\hline $\max$ & 0.12 & 0.005 & 0.005 & 0.050 & 0.60 & 0.005 & 9.5 & 0.005 & 1.20 \\
\hline & $\mathrm{Ta} \%$ & $\mathrm{~V} \%$ & $\mathrm{Nb} \%$ & $\mathrm{Cu} \%$ & $\mathrm{~B} \%$ & $\mathrm{Al} \%$ & $\mathrm{Co} \%$ & $\mathrm{~N}_{2} \%$ & Fe\%- \\
\hline $\min$ & 0.10 & 0.15 & - & - & - & - & - & 0.015 & Balance \\
\hline $\max$ & 0.14 & 0.25 & 0.001 & 0.005 & 0.001 & 0.01 & 0.005 & 0.045 & Balance- \\
\hline
\end{tabular}

References

1. F. A. Moeslang, E.Diegele, M. Klimankov, R. Laesser, R. Lindau, E. Lukon, et al Nuclear Fusion 45 (2005) 649-655

\section{PROPERTY PARAGRAPH STRUCTURE}

MATERIAL identification

PROPERTY identification

PROPERTY description

Relevant plots and tables

- Property temperature or dose evolution formula

Allowables

References 


\section{Material EUROFER}

\section{Property Yield Strength}

Table 6.3. Average and minimum yield strength for as-received EUROFER97, and allowable stress values

\begin{tabular}{|c|c|c|c|c|c|}
\hline Temperature & $\mathbf{R}_{\mathbf{p} 02 \text { average }}$ & $\mathbf{R}_{\mathrm{p} 02 \text { min }}$ & $\mathbf{S m}$ & $\mathbf{S}$ & $\mathbf{S}_{\mathrm{mB}}$ \\
\hline${ }^{\circ} \mathrm{C}$ & $\mathrm{Mpa}$ & $\mathbf{M p a}$ & $\mathrm{MPa}$ & $\mathrm{MPa}$ & $\mathrm{MPa}$ \\
\hline-200 & 1093 & 990 & & & \\
\hline-150 & 843 & 764 & & & \\
\hline-100 & 685 & 621 & & & \\
\hline-50 & 593 & 537 & & & \\
\hline 0 & 543 & 492 & & & \\
\hline 20 & 531 & 481 & 206 & 155 & 160 \\
\hline 50 & 519 & 470 & 206 & 155 & 157 \\
\hline 100 & 508 & 460 & 206 & 155 & 153 \\
\hline 150 & 503 & 455 & 206 & 155 & 152 \\
\hline 200 & 497 & 450 & 203 & 152 & 150 \\
\hline 250 & 487 & 441 & 198 & 148 & 147 \\
\hline 300 & 473 & 428 & 191 & 143 & 143 \\
\hline 350 & 454 & 411 & 182 & 137 & 137 \\
\hline 400 & 430 & 390 & 172 & 129 & 130 \\
\hline 450 & 403 & 365 & 158 & 119 & 122 \\
\hline 500 & 371 & 336 & 142 & 107 & 112 \\
\hline 550 & 332 & 300 & 124 & 93 & 100 \\
\hline 600 & 282 & 255 & 102 & 77 & 85 \\
\hline 700 & 118 & 107 & - & - & - \\
\hline
\end{tabular}

Table 6.4. Design strength allowances trend curves

\begin{tabular}{|c|c|}
\hline Design allowance & Polynom for calculation \\
\hline Average yield strength & $542.69-0.692^{*} T+0.00512^{*} T^{2}-1.926 E-5^{*} T^{3}+2.96 E-8^{*} T^{4}-1.755 E-11^{*} T^{5}$ \\
\hline Minimum yield strength & $491.5-0.627^{*} T+0.00464^{*} T^{2}-1.744 E-5^{*} T^{3}+2.68 E-8^{*} T^{4}-1.59 E-11^{*} T^{5}$ \\
\hline$S_{m}$ & $203.3+0.087^{*} T-4.2 E-4^{*} T^{2}$ \\
\hline$S$ & $152.45+0.065^{*} T-3.16 E-4^{*} T^{2}$ \\
\hline$S_{m B}$ & $156+0.022^{*} T-2.26 E-4^{*} T^{2}$ \\
\hline
\end{tabular}




\section{Material EUROFER}

\section{Property Yield Strength - Aged (irradiated) values}
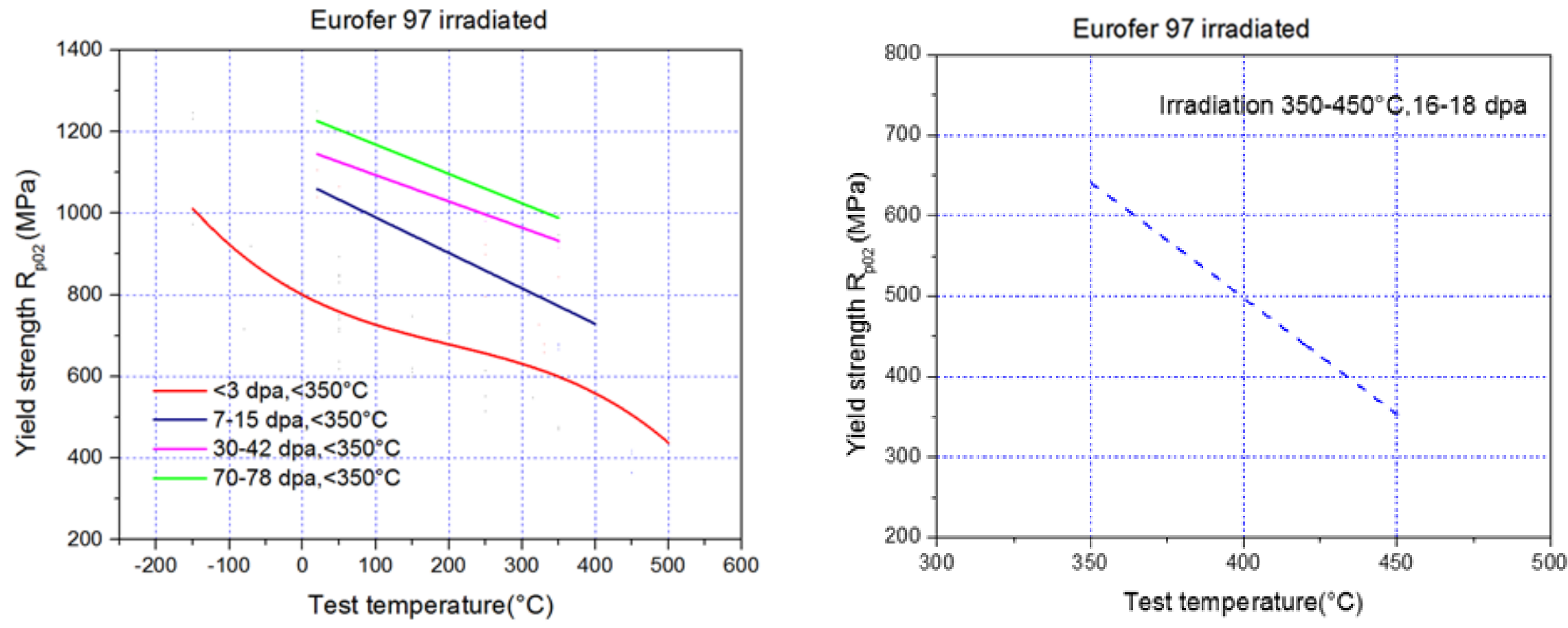

- Data grouping according to irradiation conditions

- Calculation of Yield Strength trend curves 


\section{Material EUROFER \\ Property Creep}

$S_{t}(T, t)$ defined as the least of

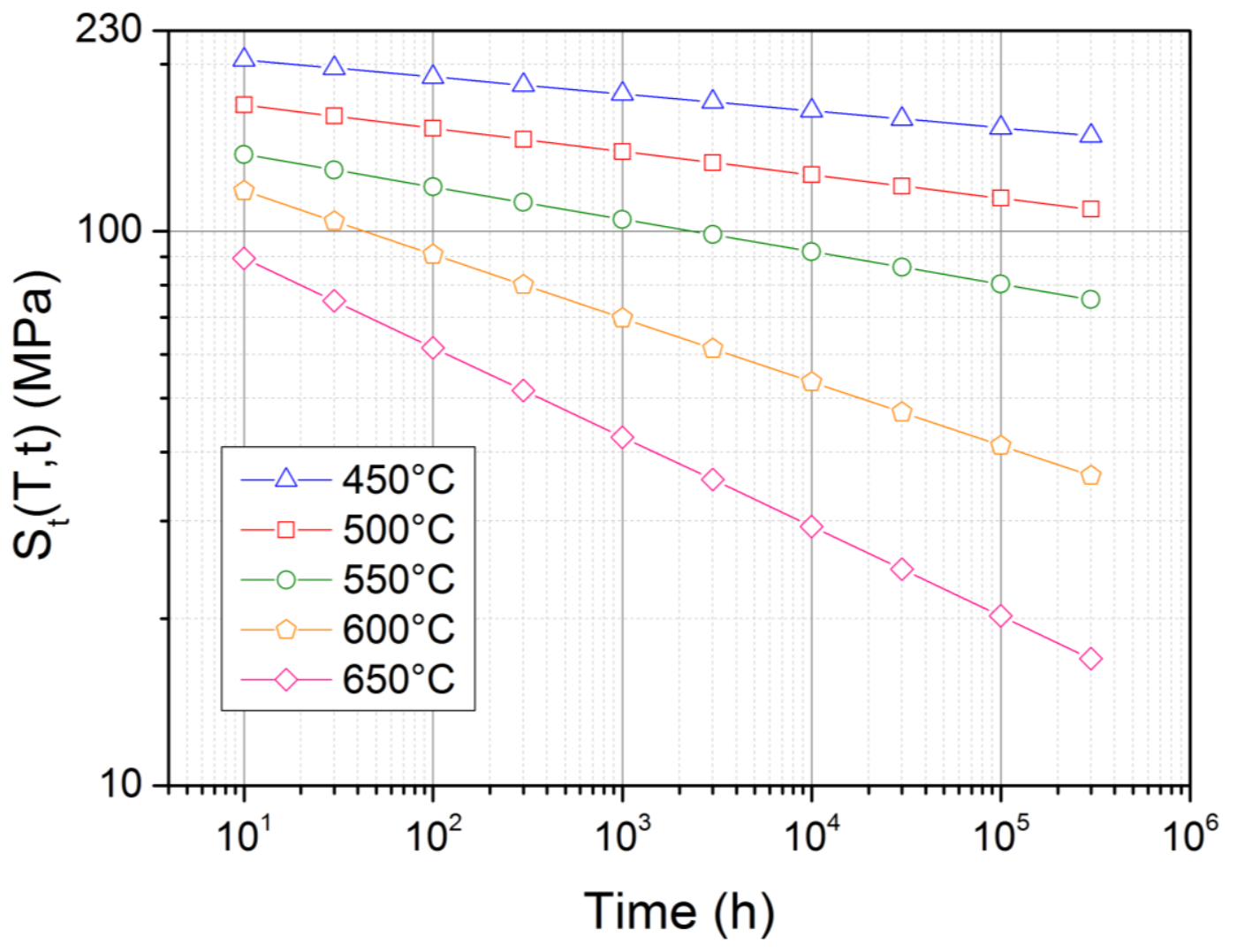

1) $2 / 3$ of the minimum stress corresponding to average creep rupture time $t$ at $T$

2) $80 \%$ of the minimum stress corresponding to time $t$ and temperature $T$ for onset of tertiary creep

3) Stress inducing total strain (elastic +plastic +creep) of $1 \%$. This stress being derived from the tensile hardening rule and from the creep strain law. A minimum value equal to 0.8 times the average value.

$S_{t}(T, t)$ are governed by $2 / 3$ of minimum stress to rupture 


\section{EUROFER97 MPH - Gaps analysis}

\section{Unirradiated}

- Fracture Mechanical (FM) properties ( $\left.\mathrm{J}_{\mid \mathrm{C}}, \mathrm{J}-\mathrm{R} \ldots\right)$

- Creep-fatigue properties

$>$ Isothermal LCF tests with long hold times under tension, compression

- Effects of cycling softening on tensile \& creep

- Ratchetting

$>$ Isothermal uniaxial ratchetting tests (stress controlled with mean stress)

$>$ Lab tests with combined primary and secondary loads

- Fatigue crack growth

$>$ Investigate the effects of R-value, temperature \& environment

Thermal aging effects (tensile, Charpy-V, FM)

- Mechanical properties on weldments for proper PWHT (tensile, LCF, FM, creep)

\section{Irradiated}

- Baseline data (tensile, LCF) in a wide temperature $\left(T_{\text {irr }}=200-550^{\circ} \mathrm{C}\right)$ and dose range

- Fracture Mechanical (FM) properties $\left(J_{\mid C}, J-R\right.$...)

- Isothermal LCF tests with hold times

- Swelling \& Helium effects

- Irradiation creep

- Mechanical properties on weldments for proper PWHT (tensile, FM) 


\section{Summary}

- EUROfusion EUROFER97 database with about 3000 records

- Application of data reviewing procedure for qualification of data for MPH

- Calculation of design allowables

- ERUFOER MPH covering over 20 DEMO design relevant properties on base metal EUROFER97

- Gaps analysis with respect to the further development of the MPH 


\section{Material Property Handbook}
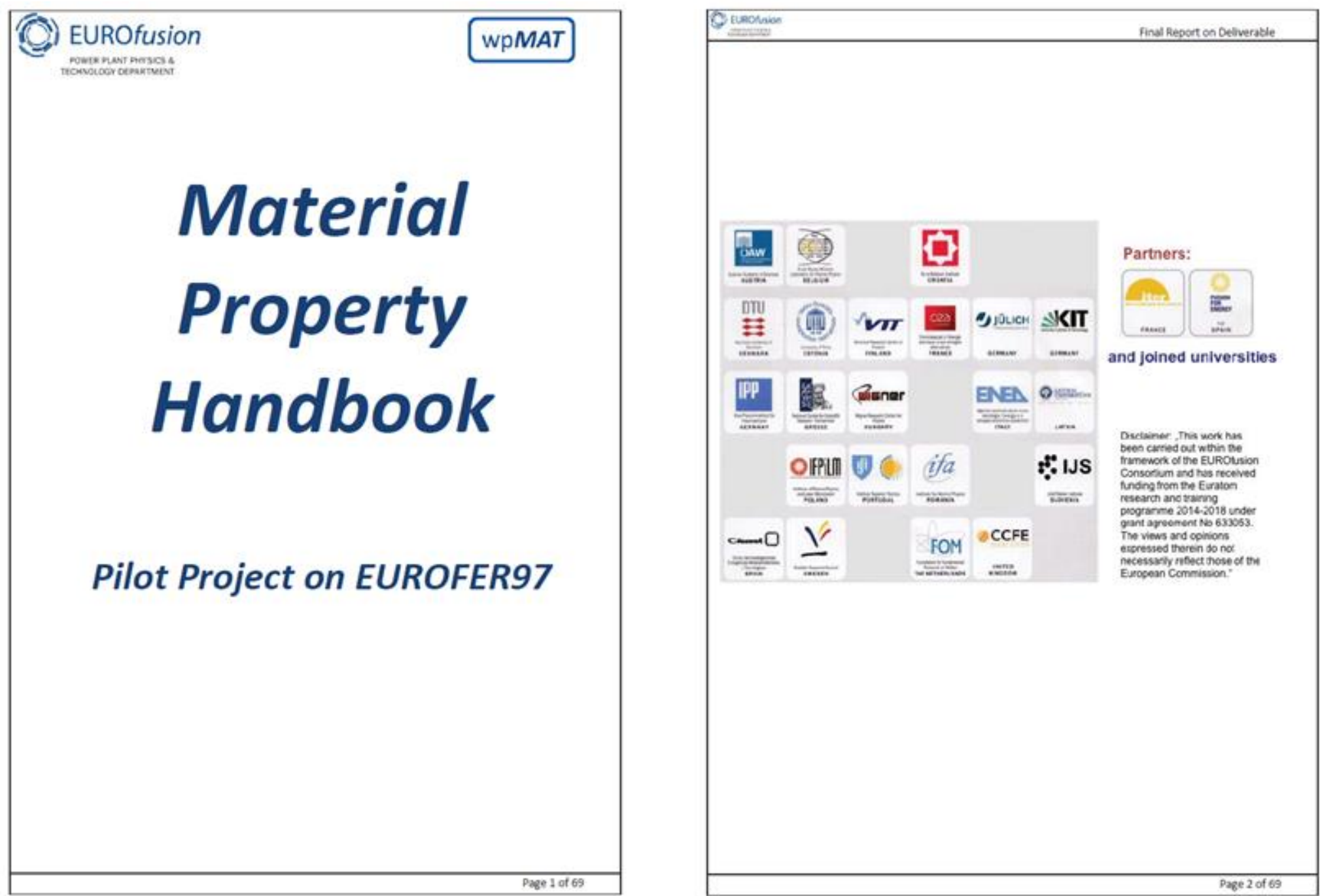